\title{
Lingual vibrotactile threshold shift during magnitude-estimation scaling: Effects on magnitude-estimation responses and scaling behavior
}

\author{
DONALD FUCCI \\ Ohio University, Athens, Ohio \\ LINDA PETROSINO \\ Bowling Green State University, Bowling Green, Ohio \\ DANIEL HARRIS \\ Healthcare Rehabilitation Center, Austin, Texas \\ and \\ ELIZABETH RANDOLPH-TYLER and SUSAN WAGNER \\ Ohio University, Athens, Ohio
}

\begin{abstract}
The purpose of the present study was to determine if lingual vibrotactile threshold shifts occurred during magnitude-estimation scaling of suprathreshold stimuli presented to the dorsal surface of the tongue. Possible relationships of the lingual vibrotactile threshold shifts to suprathreshold stimulus intensity, magnitude-estimation responses, and overall scaling behavior were explored. A single group of 24 subjects with an age range of 18 to 22 years participated in this study. Each subject performed two magnitude-estimation tasks. In one of the tasks, threshold of sensitivity was determined after every suprathreshold numerical response of the subject. If a threshold shift was recorded, threshold was allowed to return to the pretest baseline level before continuing to the next suprathreshold stimulus presentation. The results showed that threshold shift did occur during lingual vibrotactile magnitude-estimation scaling, and that it was related to suprathreshold stimulus intensity. The results also showed that the numerical magnitude-estimation responses of the subjects were different for the two scaling tasks. Overall scaling behavior of the subjects in the form of power-function exponents was not different for the two tasks.
\end{abstract}

Recent advancements in the psychophysical study of the tactile sensory system have enabled behavioral scientists to study tactile sensory-system function at both threshold and suprathreshold levels. Threshold investigation provides information about the sensitivity of the receptor mechanism, but it does not lead to information about the total range of tactile sensory function. Suprathreshold study of the tactile system is necessary to determine the behavior of the tactile sense above the peripheral level of detectability. Suprathreshold responses to tactile stimulation require higher level activity which would involve integration and association of afferent information.

In an attempt to advance the knowledge of lingual tactile sensory-system function beyond the threshold of sensitivity level, a number of studies were initiated to investigate suprathreshold subjective scaling of vibrotactile stimuli applied to the dorsum of the tongue (Fucci, Harris, \& Petrosino, 1985; Fucci, Harris, Petrosino, \& McMath,

Reprint requests may be addressed to Donald Fucci, School of Hearing \& Speech Sciences, Ohio University, Athens, OH 45701.
1987; Fucci, Petrosino, Harris, \& McMath, 1986; Fucci, Petrosino, Harris, \& Randolph-Tyler, 1987; Fucci, Petrosino, Harris, \& Randolph-Tyler, 1988; Petrosino, Fucci, Harris, \& Ellis, 1988; Petrosino, Fucci, Harris, \& Randolph-Tyler, 1988). These studies were designed to examine sensory processes operating during the scaling of tactile stimuli presented at controlled levels above threshold. The primary scaling mode utilized was magnitude estimation (Gescheider, 1985; Stevens, 1955). Lingual vibrotactile magnitude estimation requires subjects to assign numbers to various suprathreshold intensity levels of a vibratory stimulus presented to the anterior dorsum of the tongue.

Threshold and suprathreshold levels of sensory processing have been, for the most part, considered separately in lingual vibrotactile research. Fucci et al. (1985) performed a study in which correlation coefficients were calculated for lingual vibrotactile thresholds and suprathreshold magnitude-function exponents for 26 adult subjects. No significant correlations were found for the threshold data and suprathreshold exponents, suggesting 
that the two levels of processing function independently of each other with regard to the lingual tactile sense. The Fucci et al. study was designed to utilize thresholds which were obtained before suprathreshold scaling was initiated. It did not look at possible integrative processes that might cause threshold changes or fluctuations to occur during the suprathreshold scaling procedure, or influences that these threshold changes might have on scaling results. The purpose of the present study was to extend present knowledge of lingual tactile sensory-system function by determining if lingual vibrotactile threshold shifts occurred during magnitude-estimation scaling of suprathreshold stimuli presented to the dorsal surface of the tongue. Possible relationships of the lingual vibrotactile threshold shifts to suprathreshold stimulus intensity, magnitude-estimation responses, and overall scaling behavior were explored.

\section{METHOD}

\section{Subjects}

A single group of 24 subjects participated in this study. The subjects ranged in age from 18 to 22 years (mean $=19.5$ years). All subjects had normal speech and hearing and were screened (by interview) for the presence of any medical or physical conditions that could have interfered with test results. None of the subjects had prior experience with vibrotactile testing procedures.

\section{Apparatus}

The vibrotactile stimulus-control unit included a sine-wave generator, an experimenter-controlled variable attenuator, a rise/fall gate, two universal timers, an audio amplifier, a power amplifier, and an electromagnetic minivibrator with a probe-contactor extension. The pulsed vibratory signal generated had a frequency of $250 \mathrm{~Hz}$, a $50 \%$ duty cycle (on $500 \mathrm{msec}$ and off $500 \mathrm{msec}$ ) and a rise-fall time of 50 msec. The vibrotactile stimulus-measurement unit included an accelerometer, a cathode follower, a microphone amplifier, and a voltmeter. The auditory-masking unit consisted of a masking generator and TDH-49P headphones. It was used to present a narrow band of noise centered around $250 \mathrm{~Hz}$ at $70 \mathrm{~dB}$ HTL bilaterally. It has been shown that auditory masking is not a critical part of the vibrotactile testing procedure in that similar lingual vibrotactile threshold values and magnitude-estimation power-function exponents can be obtained under conditions of masking and no masking (Petrosino, Fucci, Harris, \& Ellis, 1988). The subjects appeared to prefer the masking by indicating that they could concentrate better on the vibratory signals being delivered to the tongue. The masking was set at a reasonable intensity level ( $70 \mathrm{~dB}$ HTL) so that it did not become a distractor. The higher intensity vibratory signals being delivered to the tongue were somewhat audible to the subjects during testing. A detailed description of the vibrotactile equipment and procedures can be found in a review by Harris, Fucci, Petrosino, and Wallace (1986).

\section{Procedure}

Each subject completed a series of two lingual vibrotactile magnitude-estimation scaling tasks, which were conducted in separate test sessions scheduled 1 week apart (Stevens, 1955). For both scaling tasks, the subject was seated in an adjustable chair and positioned so that the tongue could be placed against the bottom of a rigidly mounted plastic disk. A hole in the center of the disk provided access for the probe-contactor extension of the vibrator to the anterior midline section of the dorsum of the tongue. The contactor on the end of the probe had an area of $.128 \mathrm{~cm}^{2}$, and there was a 1-mm gap between the contactor and the disk. The TDH-49P headphones were placed over the subject's ears for binaural auditory masking of the $250-\mathrm{Hz}$ vibrotactile stimulus being applied to the tongue.

An ascending method of limits was used to obtain thresholds of sensitivity for all subjects prior to each of the two magnitudeestimation tasks (Hall, Fucci, \& Arnst, 1972). This method of threshold testing was chosen over the forced-choice criterion-free method of threshold testing to minimize subject fatigue and sensorysystem adaptation (Petrosino \& Fucci, 1983). Accepted threshold was the mean of three successive readings within a $5-\mathrm{mV}$ range (Telage \& Fucci, 1974). These obtained thresholds were necessary so that the suprathreshold intensities used for magnitude estimation could be set in reference to each subject's threshold of sensitivity (Verrillo, Fraioli, \& Smith, 1969). They were also necessary in this experiment for determination of threshold shift and for the return of threshold to baseline in the second magnitude-estimation scaling task.

During the first lingual vibrotactile magnitude-estimation task, each subject was required to assign numbers to a series of pulsed, $250-\mathrm{Hz}$ vibrotactile stimuli at eight randomly presented stimulusintensity levels, ranging from 6 to $40 \mathrm{~dB} S \mathrm{SL}(6,10,16,20,26$, $30,36$, and $40 \mathrm{~dB} \mathrm{SL})$. The subject was allowed to feel the pulsed, $250-\mathrm{Hz}$ stimulus on the tongue for a maximum of $15 \mathrm{sec}$ before being required to provide a numerical response. The subject was instructed to think of a number that matched the strength of the vibration felt on the tongue. Whole numbers, decimals, and fractions were indicated as permissible selections (Zwislocki \& Goodman, 1980). The subjects were encouraged to be spontaneous in selecting numbers and to make judgments at each stimulus-intensity level without reference to those previously presented. After each recording of the subject's verbal numerical response, the tongue was realigned with the probe-contactor extension of the vibrator in preparation for the subsequent stimulus presentation. The geometric means of the 24 subjects' numerical responses to a single run of each of the eight stimulus-intensity levels were taken as the mean lingual vibrotactile magnitude-estimation responses for the first scaling task (Petrosino, Fucci, \& Harris, 1985).

During the second scaling task, lingual vibrotactile magnitudeestimation scaling was conducted in a manner similar to that described for the first scaling task, with two procedural changes. First, immediately after the subject provided his/her verbal numerical response to the 15-sec presentation of the pulsed, $250-\mathrm{Hz}$ stimulus at one of the eight intensity levels, lingual vibrotactile threshold of sensitivity was rechecked using the threshold technique described above. This post-magnitude-estimation-response threshold determination allowed the experimenter to assess threshold shift resulting from the suprathreshold stimulus presentation. Second, if threshold shift was detected, the experimenter performed subsequent threshold checks at 30 -sec intervals until threshold returned to the prescaling baseline-sensitivity level, before continuing to the next suprathreshold stimulus presentation. In this manner, the experimenter was assured that each subsequent suprathreshold stimulus presentation was initiated with the subject's threshold at the prescaling baseline level. Post-magnitude-estimation-response threshold determinations were conducted for each of the eight randomly presented suprathreshold stimulus presentations. Lingual vibrotactile threshold shifts for each subject at each of the eight suprathreshold stimulus-intensity levels were recorded in decibels sensation level and averaged across the 24 subjects. The geometric means of the 24 subjects' numerical responses to a single run of each of the eight stimulus-intensity levels were taken as the mean lingual vibrotactile magnitude-estimation responses for the second scaling task (Petrosino et al., 1985). 
A counterbalanced technique was utilized to control for possible learning effects with regard to the lingual vibrotactile scaling tasks. Twelve of the subjects performed the first scaling task during the first test session and the second scaling task during the second test session conducted 1 week later. The remaining 12 subjects performed the second scaling task during the first test session and the first scaling task during the second test session conducted 1 week later.

\section{RESULTS}

It can be seen in Figure 1 that threshold shift did occur for each of the eight suprathreshold stimulus-intensity levels employed in the second scaling task, and that the amount of shift appeared to increase as stimulus intensity was increased. A repeated measures analysis of variance (ANOVA; Barcikowski, 1983; Bock, 1975) revealed a significant effect of suprathreshold stimulus intensity on lingual vibrotactile threshold shift $(p \leq .01)$.

A repeated measures ANOVA revealed a significant difference $(p \leq .01)$ between the numerical responses of the subjects in the first and second scaling tasks.

The average time required for threshold to return to baseline levels between trials in the second scaling task was not a factor considered to be part of the stated purpose of this study. Informal observation of this parameter provided mean estimates of $2.5 \mathrm{~min}$ for threshold to return to baseline for the lowest intensity level tested (6 dB SL) and $8.5 \mathrm{~min}$ for threshold to return to baseline for the highest intensity level tested (40 dB SL).

Lower and upper power-function exponents were derived for each subject for the two scaling tasks employed in this experiment. The exponents are reported in terms of intensity (Verrillo \& Chamberlain, 1972). It appears that lingual vibrotactile magnitude-estimation scales may show a limitation in magnitude-growth functions around and above 25 dB SL. Harper and Stevens (1964) hypothesized that for certain continua, an "upper threshold" may

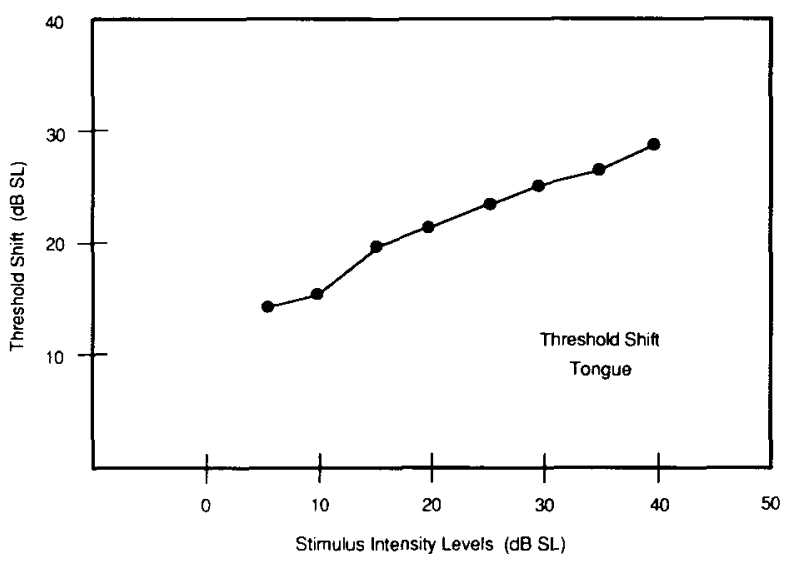

Figure 1. Threshold shift on the tongue as a function of suprathreshold stimulus intensity. exist, and that above this region, increases in stimulus values show no corresponding gradations in subjective magnitude. The present magnitude-estimation data for both scaling tasks reflected such an asymptotic growth function and are consistent with previous research (Fucci, Harris, \& Petrosino, 1984; Petrosino, Fucci, \& Harris, $1985,1987)$. The mean exponents for the lower functions were .58 for both scaling tasks, and the mean exponents for the upper functions were .17 for the first scaling task and .20 for the second scaling task.

The lower and upper power-function exponents derived for each subject for the two scaling tasks were compared using a $t$ test for related measures (Bruning \& Kintz, 1977). No significant difference $(p \leq .01)$ was found between the subjects' lower and upper power-function exponents for the two scaling tasks involved in this experiment. The positions of the plotted power functions were also very similar for both scaling tasks, adding further support to the statistical findings.

\section{DISCUSSION}

The results of this study show that threshold shift can occur during lingual vibrotactile magnitude-estimation scaling when a $250-\mathrm{Hz}$ stimulus of $15-\mathrm{sec}$ duration is employed. They further show that the amount of threshold shift is directly related to suprathreshold stimulus intensity (Figure 1).

The numerical responses of the subjects to the suprathreshold stimulus intensities appear to have been influenced by the threshold shifts that occurred during the magnitude-estimation scaling tasks. The subjects consistently provided higher numerical responses at each of the eight suprathreshold intensity levels for the task in which threshold was allowed to return to prescaling baseline than they did for the task in which possible threshold shift was not determined or controlled for during the scaling procedure. It is difficult at this time to reflect on why the subjects consistently presented larger numerical responses when threshold was allowed to return to baseline levels before each suprathreshold stimulus presentation. It could be that the subjects were using threshold of sensitivity as a reference for the their scaling behavior, and that they used this reference differently when it was free to vary during scaling as opposed to when it was controlled and set at baseline levels prior to each suprathreshold stimulus presentation. A reference standard was deliberately not employed in the scaling procedure (Hellman \& Zwislocki, 1963), but informal questioning of the subjects after testing was completed indicated that most of them had developed an internal referent of their own for scaling purposes. The most likely chosen referent related to what the subjects imagined was the weakest vibratory tongue sensation that they could feel.

Fucci et al. (1984), using magnitude-estimation scaling, reported larger magnitude-scaling responses when 
vibrotactile stimuli were presented to the thenar eminence of the hand as opposed to the dorsum of the tongue. The differences in the magnitude-scaling responses for the two different test sites were probably related to the inherent differences in the makeup of the sites, which also have an effect on their vibrotactile thresholds of sensitivity (Verrillo, 1966). The mean threshold for the hand is approximately $-10 \mathrm{~dB}$ re 1 micron, and the mean threshold for the lingual dorsum is approximately $4 \mathrm{~dB}$ re 1 micron (Fucci et al., 1988). In the present study, a similar "threshold effect" might have occurred in that when threshold was allowed to return to baseline before each new suprathreshold stimulus presentation, it would have been at a lower intensity level than would have been the case if a threshold shift had taken place and scaling were continued with that shift carried over to the next stimulus presentation. The more sensitive thresholds for the thenar eminence appear to be related to higher numerical responses, and the same kind of effect might have been occurring in the present study when threshold shift was controlled for.

The similar power-function exponents derived for the two magnitude-estimation scaling tasks suggest that overall scaling behavior was not affected by threshold manipulations. In both tasks, the subjects used approximately the same scaling range, and the same type of limitations in magnitude-growth functions were noted around $25 \mathrm{~dB} \mathrm{SL}$. It could be that overall scaling behavior is reflective of an internal, absolute scaling mechanism which is established early in life, is very stable, and is not affected by tactile functioning at the sensory-receptor level (Fucci et al., 1985; Zwislocki \& Goodman, 1980).

The findings of this study would lead to the suggestion that researchers involved in magnitude-estimation scaling research, with controlled vibration as the stimulus, should be aware that there is possible threshold shifting going on during the scaling task, and that this peripheral receptor activity could have an effect on the numerical responses of the subjects, even though it does not seem to relate to overall scaling behavior in terms of scaling range and power-function exponents derived from the data. Future studies should be undertaken in which the vibratory stimulus parameters of frequency, intensity, and duration are studied as to their integrative effects on threshold activity during magnitude-estimation scaling. Nonoral test sites should also be included to determine if threshold shift during magnitude-estimation scaling is a wide-spread phenomenon or an activity to be found only within the oral region.

\section{REFERENCES}

BARCIKOWSKI, R. (1983). Computer packages and research design: Vol. 2. SAS. New York: University Press of America.

Bock, R. (1975). Multivariate statistical methods in behavioral research. New York: McGraw-Hill.

Bruning, J., \& KINTZ, B. (1977). Computational handbook of statistics. Glenview, IL: Scott, Foresman.

Fucci, D., Harris, D., \& Petrosino, L. (1984). Sensation magnitude scales for vibrotactile stimulation of the tongue and thenar eminence. Journal of Perceptual \& Motor Skills, 58, 843-848.

Fucci, D., Harris, D., \& Petrosino, L. (1985). Threshold and suprathreshold correlations for the oral tactile sensory mechanism. Journal of Speech \& Hearing Research, 28, 331-335.

Fucci, D., Harris, D., Petrosino, L., McMath, E. (1987). The effects of psychophysical scaling method, body test site, and skin contactor surface area on vibrotactile magnitude. Journal of Perceptual \& Motor Skills, 64, 1127-1138.

Fucci, D., Petrosino, L., Harris, D., \& McMath, E. (1986). Stimulus duration effects on vibrotactile magnitude estimation for the tongue and hand. Bulletin of the Psychonomic Society, 24, 193-196.

Fucci, D. Petrosino, L., Harris, D., \& RandolPh-Tyler, E. (1987). Effects of aging on responses to suprathreshold lingual vibrotactile stimulation. Journal of Perceptual \& Motor Skilks, 64, 683-694.

Fucci, D., Petrosino, L., Harris, D., \& RandolPH-Tyler, E. (1988). Exposure effects on the psychophysical scaling methods of magnitude estimation and cross-modal matching for vibrotactile stimulation of the tongue and hand. Journal of Perceptual \& Motor Skills, 66, 479-485.

GescheIDER, G. A. (1985). Psychophysics: Method, theory, and application. New York: Wiley.

HALL, D., FuCCI, D., \& ARNST, D. (1972). Vibrotactile stimulation: An investigation of psychophysical methods for establishing threshold. Journal of Perceptual \& Motor Skills, 34, 891-898.

HARPER, R., \& STEVENS, S. S. (1964). Subjective hardness of compliant materials. Quarterly Journal of Experimental Psychology, 16, 204-215.

Harris, D., Fucci, D., Petrosino, L., W Wallace, L. (1986). Instrumentation for magnitude estimation and cross-modality matching of auditory and lingual vibrotactile sensations. Review of Scientific Instruments, 57, 2343-2347.

Hellman, R. P., \& ZWISLOCKI, J. (1963). Monaural loudness function at $1000 \mathrm{cps}$ and interaural summation. Journal of the Acoustical Society of America, 35, 856-865.

Petrosino, L., \& Fucci, D. (1983). A precision method for lingual vibrotactile threshold measurement. Bulletin of the Psychonomic Society, 21, 203-205.

Petrosino, L., Fucci, D., \& Harris, D. (1985). Effects of singlesession repetitive judgments on magnitude estimation scales for lingual vibrotactile sensation. Perception \& Psychophysics, 37, 205-208.

Petrosino., L., Fucci, D., \& Harris, D. (1987). Magnitude estimation and magnitude production: Stimulus frequency effects on magnitudes of lingual vibrotactile sensation. Journal of Perceptual \& Motor Skills, 64, 663-670.

Petrosino, L., Fucci, D., Harris, D., \& Ellis, L. (1988). Effect of auditory masking on lingual vibrotactile thresholds and magnitude estimation scaling responses. Journal of Perceptual \& Motor Skills, 67, 491-494.

Petrosino, L., Fuoci, D., Harris, D., \& Randolph-Tyler, E. (1988). Lingual vibrotactile/auditory magnitude estimation and cross-modal matching: Comparison of suprathreshold responses in men and women. Journal of Perceptual \& Motor Skills, 67, 291-300.

STEvens, S. S. (1955). The measurement of loudness. Journal of the Acoustical Society of America, 27, 815-820.

Telage, K., \& FuccI, D. (1974). Concerning intrasubject measurements of successive lingual vibrotactile responses. Joumal of Perceptual \& Motor Skills, 39, 1047-1052.

VERRILlo, R. T. (1966). Specificity of a cutaneous receptor. Perception \& Psychophysics, 1, 149-153.

Verrillo, R. T., \& Chamberlain, S. C. (1972). The effect of neural density and contactor surround on vibrotactile sensation magnitude. Perception \& Psychophysics, 11, 117-120.

Verrillo, R. T., Fraioli, A. J., \& SMith, R. L. (1969). Sensation magnitude of vibrotactile stimuli. Perception \& Psychophysics, 6, 366-372.

ZWISLOCKI, J. J., \&OODMan, D. A. (1980). Absolute scaling of sensory magnitudes: A validation. Perception \& Psychophysics, 28, 28-38.

(Manuscript received September 26, 1988; revision accepted for publication March 23, 1989.) 